\title{
Acolhimento na Estratégia Saúde da Família: perspectivas das pessoas com deficiência no contexto rural
}

Welcoming in the Family Health Strategy: perspectives of people with disabilities in the rural context

Acogimiento en la Estrategia de Salud Familiar: perspectivas de las personas con discapacidad en el contexto rural

\section{Taís Cristiane Lenz ${ }^{\mathrm{I}}$, Marta Cocco da Costa ${ }^{\mathrm{II}}$, Isabel Cristina dos Santos Colomé ${ }^{\mathrm{III}}$, Andressa de Andrade ${ }^{\mathrm{IV}}$, Neila Santini de Souzav ${ }^{\mathrm{v}}$, Jaqueline Arboit ${ }^{\mathrm{VI}}$}

\begin{abstract}
Resumo: Objetivo: analisar as percepções das pessoas com deficiência física que residem no contexto rural acerca do acolhimento prestado pelas equipes da Estratégia Saúde da Família. Método: estudo qualitativo, desenvolvido em seis municípios do Rio Grande do Sul, Brasil, cujos participantes foram treze pessoas com deficiência física. A produção das informações ocorreu de janeiro a maio de 2019 por entrevistas semiestruturadas, analisadas pela modalidade temática. Resultados: o acolhimento foi relacionado ao carinho, afeto, relação de confiança, diálogo e proximidade com os profissionais, bem como à resolutividade das demandas ou encaminhamento para outros serviços. Contudo, elencaram dificuldades quanto à disponibilidade de medicações, exames, atendimento médico e agendamento do atendimento. Conclusão: apesar do esforço dos profissionais das equipes em acolher as pessoas com deficiência física residentes no contexto rural e atender suas demandas, revela-se a centralização do cuidado nas questões biológicas e as limitações de acessibilidade e acesso aos serviços de saúde.
\end{abstract}

Descritores: Pessoas com deficiência; Zona rural; Estratégia saúde da família; Acolhimento; Enfermagem

\footnotetext{
${ }^{\text {I }}$ Enfermeira, Pós-graduanda em Saúde Pública com ênfase em Saúde da Família, Centro Universitário Internacional (UNINTER), Santa Rosa, RS, Brasil. E-mail: taislenz123@gmail.com ORCID: https://orcid.org/0000-0003-3425-9523

II Enfermeira, Doutora em Enfermagem, Universidade Federal de Santa Maria (UFSM)/Docente do Departamento de Ciências da Saúde, Palmeira das Missões, RS, Brasil. E-mail: marta.c.c@ufsm.br ORCID: https://orcid.org/0000-0002-9204-3213

III Enfermeira, Doutora em Enfermagem, Universidade Federal de Santa Maria (UFSM)/Docente do Departamento de Ciências da Saúde, Palmeira das Missões, RS, Brasil. E-mail: enfbel@yahoo.com.br ORCID: https://orcid.org/0000-0001-7680-3289

IV Enfermeira, Doutora em Ciências da Saúde, Universidade Federal de Santa Maria (UFSM)/Docente do Departamento de Ciências da Saúde, Palmeira das Missões, RS, Brasil. E-mail: andressa@ufsm.br ORCID: https://orcid.org/0000-0002-5977-409X

${ }^{\vee}$ Enfermeira, Doutora em Ciências, Universidade Federal de Santa Maria (UFSM)/Docente do Departamento de Ciências da Saúde, Palmeira das Missões, RS, Brasil. E-mail: neilasantini25@gmail.com ORCID: https://orcid.org//0000-0002-5083-9432

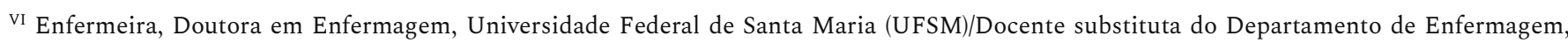
Santa Maria, RS, Brasil. E-mail: jaqueline.arboit@hotmail.com ORCID: https://orcid.org/0000-0002-6610-5900
} 
Abstract: Objective: to analyze the perceptions of people with physical disabilities living in the rural context about the welcoming provided by the Family Health Strategy teams. Method: qualitative study, developed in six cities in Rio Grande do Sul, Brazil, whose participants were 13 people with physical disabilities. The production of information took place from January to May 2019 through semi-structured interviews, analyzed by thematic modality. Results: welcoming was related to fondness, affection, relationship of trust, dialogue and closeness with professionals, as well as resolvability of demands or referral to other services. Nevertheless, they listed difficulties regarding the availability of medications, exams, medical care and scheduling of care. Conclusion: despite the efforts of the professionals working in the teams to welcome people with physical disabilities living in the rural context and meet their demands, the centralization of care in biological issues and the limitations of accessibility and access to health services are revealed.

Descriptors: Disabled Persons; Rural Areas; Family Health Strategy; User Embracement; Nursing.

Resumen: Objetivo: analizar las percepciones de las personas con discapacidad física que viven en el contexto rural sobre la acogida brindada por los equipos de la Estrategia de Salud Familiar. Método: estudio cualitativo, desarrollado en seis ayuntamientos de Rio Grande do Sul, Brasil, cuyos participantes fueron 13 personas con discapacidad física. La producción de la información tuvo lugar de enero a mayo de 2019 mediante entrevistas semiestructuradas, analizadas por modalidad temática. Resultados: el acogimiento se relacionó con el cariño, el afecto, la relación de confianza, el diálogo y la cercanía con los profesionales, así como la resolución de demandas o la referencia a otros servicios. Sin embargo, enumeraron dificultades con respecto a la disponibilidad de medicamentos, exámenes, atención médica y programación de la atención. Conclusión: a pesar del esfuerzo de los profesionales de estos equipos para acoger a las personas con discapacidad física que viven en el contexto rural y atender sus demandas, se revela la centralización de la atención en cuestiones biológicas y las limitaciones de accesibilidad y acceso a los servicios de salud.

Descriptores: Personas con discapacidad; Medio rural; Estrategia de salud familiar; Acogimiento; Enfermería

\section{Introdução}

O conceito de deficiência ao longo da história obteve variados direcionamentos, sendo influenciado por aspectos políticos, éticos e filosóficos. ${ }^{1}$ Atualmente, tem-se o modelo social de deficiência como o mais aceito para explicar esta condição. Este modelo busca o rompimento da visão biológica e positivista que o modelo médico adota, limitando a compreensão de deficiência às lesões e impedimentos do corpo, bem como o desenvolvimento de políticas públicas que não sejam direcionadas unicamente aos aspectos biológicos dessas pessoas. ${ }^{2}$

Nessa direção, pessoas com deficiência (PCDs) são aquelas que apresentam impedimentos físicos, mentais, intelectuais ou sensoriais de longo prazo, os quais ao interagir com diferentes barreiras tendem a limitar a sua inclusão plena em todas as esferas da vida social 
3 | Lenz TC, Costa MC, Colomé ICS, Andrade A, Souza NS, Arboit J

em condições de igualdade com os demais indivíduos. O estudo em tela centra-se nas perspectivas de PCD física, cuja participação na sociedade é limitada devido a alterações completas ou parciais de um ou mais segmentos do corpo, ocasionando perda ou anormalidade de estrutura ou função de membro. ${ }^{3}$

A Política Nacional de Saúde da Pessoa Portadora de Deficiência conta, dentre as suas diretrizes, com a promoção da qualidade de vida e organização dos serviços de atenção à PCD, instrumentalização de recursos humanos e assistência integral à saúde dessa população. ${ }^{3}$ Apesar das recomendações desta política, existem inúmeros obstáculos sociais, econômicos e culturais, vivenciados por essas pessoas, para sua inserção plena na sociedade, ${ }^{4}$ incluindo o acesso aos serviços de atenção à saúde.

As barreiras vivenciadas pelas PCDs são agravadas quando estas residem em contextos rurais. Nestes, a população apresenta menor nível de escolaridade, baixa renda e acesso dificultado aos serviços de saúde devido à distância geográfica dos centros urbanos, além da baixa cobertura assistencial da rede de atenção à saúde, o que implica negativamente em sua qualidade de vida. ${ }^{5}$

No que se refere ao acesso aos serviços de saúde no contexto rural, a Atenção Primária à Saúde (APS) é o serviço que está presente na maioria dos municípios brasileiros. A Estratégia Saúde da Família (ESF) se constitui como porta de entrada aos demais níveis de atenção à saúde, o que se torna ainda mais evidente no contexto rural. Ao se considerar a interface entre a saúde de PCDs e o acesso aos serviços da APS, é relevante citar que as PCDs são mais propensas a apresentar condições de déficit de saúde, sejam elas demandas físicas ou psicológicas, que requerem mais estes serviços do que pessoas sem deficiência. ${ }^{6}$

O trabalho cotidiano das equipes de ESF busca a resolução das demandas e queixas da população adstrita. Seu foco de atenção e cuidado está centrado nas famílias e coletividades, visando à prevenção e à promoção da saúde, além do atendimento clínico. O nível de resolutividade da ESF está intimamente relacionado ao acolhimento e vínculo na relação entre o profissional e usuário. ${ }^{7}$ 
Acolhimento na Estratégia Saúde da Família: perspectivas das pessoas com deficiência...| 4

O acolhimento é uma das principais diretrizes da Política Nacional de Humanização do Sistema Único de Saúde (SUS) no Brasil. É definido como a recepção do usuário no serviço e responsabilização dos profissionais por este usuário, a partir da escuta de necessidades e angústias. Busca a garantia de uma assistência resolutiva e a articulação com os outros serviços de saúde para a continuidade do cuidado, sempre que necessário. ${ }^{8}$

O acolhimento proporciona uma relação melhor entre usuários e profissionais de saúde, fomentada dentre outros, pelo afeto, posturas acolhedoras e respeito. Ao acolher e escutar o usuário, proporcionam-se o estabelecimento e fortalecimento de vínculo fundamental à humanização do cuidado e resolutividade da demanda apresentada pelo usuário e família. ${ }^{9}$ Trata-se de uma tecnologia que busca qualificar a escuta e diminuir a fragmentação do cuidado prestado. ${ }^{10}$

Em vista dos desafios do setor da saúde em construir ações de cuidado às PCDs que residem no contexto rural, em especial, o acolhimento, o presente estudo teve como questão de pesquisa: quais as percepções das pessoas com deficiência física que residem no contexto rural acerca do acolhimento prestado pelas equipes da Estratégia Saúde da Família? Para respondê-la tem-se como objetivo analisar as percepções das pessoas com deficiência física que residem no contexto rural acerca do acolhimento prestado pelas equipes da Estratégia Saúde da Família.

\section{Método}

Trata-se de um estudo descritivo de abordagem qualitativa. Esta é empregada em investigações que buscam a história, relações, representações, crenças, percepções e opiniões do ser humano acerca de si mesmo, de suas formas de viver e construir seus artefatos, bem como seus sentimentos e pensamentos. ${ }^{11}$

O cenário de estudo constituiu-se de seis municípios das regiões norte e noroeste do Rio

Grande do Sul, Brasil, pertencentes à 15a e 19ª Coordenadorias Regionais de Saúde. A escolha desses municípios justifica-se por serem de pequeno porte populacional e $70 \%$ de sua população 
5 | Lenz TC, Costa MC, Colomé ICS, Andrade A, Souza NS, Arboit J

residir em contexto rural. Nestes municípios havia poucas informações quanto ao número de pessoas com deficiência residentes na área rural, apesar da evidência de existência dessa população. Pontua-se que o número total de unidades de ESF nos municípios cenário do estudo era de oito. Em quatro destes municípios as unidades de ESF não se localizavam no contexto rural.

Para a organização e produção das informações, primeiramente foi realizado contato com as enfermeiras coordenadoras de todas as unidades de ESF dos municípios cenário do estudo. Estas, juntamente com os agentes comunitários de saúde (ACS) elaboraram uma lista com 28 possíveis participantes do estudo, ou seja, a indicação ocorreu pelas enfermeiras e ACS dos municípios. Os critérios de inclusão elencados para esta pesquisa foram: possuir deficiência física congênita ou adquirida; demonstrar condições cognitivas para responder a entrevista; ser maior de 18 anos e residir em contexto rural. O critério de exclusão estabelecido foi: possuir alguma deficiência intelectual, mental ou sensorial. Foram excluídos dois possíveis participantes, pois possuíam, simultaneamente, deficiência física e intelectual. Assim, com base nos critérios, participaram da pesquisa 13 PCDs. Destaca-se que nenhum dos participantes convidados se recusou a participar do estudo.

Os ACS da microárea de adscrição dos participantes realizou o agendamento prévio da entrevista com estes, assim como acompanhou a pesquisadora responsável até o seu domicílio para a realização da produção das informações. Ao chegar ao domicílio e verificar a presença de outras pessoas, especialmente de vizinhos, a produção das informações era reagendada. Ainda, cita-se que nem ACS nem familiares das PCDs permaneceram junto a estas durante a produção das informações. Estes procedimentos buscaram reduzir a possibilidade de vieses no estudo, bem como não ferir os princípios éticos de pesquisa.

A produção das informações ocorreu mediante entrevista semiestruturada a partir de um roteiro contendo perguntas fechadas em relação às características sociodemográficas dos participantes e questões abertas relacionadas às suas percepções sobre acolhimento, como este 
Acolhimento na Estratégia Saúde da Família: perspectivas das pessoas com deficiência... 6

se dava pela equipe da ESF, dificuldades e potencialidades percebidas quanto ao acolhimento. As entrevistas tiveram duração média de 25 minutos. As informações foram audiogravadas com o consentimento dos participantes, assegurando a sua fidedignidade. O período de produção das informações foi de janeiro a maio de 2019 , sendo encerrado quando foi compreendida a lógica interna do objeto de estudo. ${ }^{12}$

Para sistematização e análise das informações, optou-se pela análise de conteúdo temática, percorrendo as seguintes etapas: pré-análise, exploração do material e tratamento dos resultados obtidos e interpretação. $\mathrm{Na}$ pré-análise, realizaram-se, a escuta das entrevistas, leitura flutuante e constituição do corpus de acordo com objetivo do estudo. Na exploração do material, ocorreu a codificação por meio das palavras ou frases semelhantes nos discursos, permitindo a constituição dos núcleos de sentido, os quais posteriormente foram agrupados e possibilitaram a constituição das categorias. No tratamento dos resultados e interpretação, foram realizadas as inferências e interpretações com base na literatura nacional e internacional. ${ }^{11} \mathrm{~A}$ partir da análise, emergiram duas categorias temáticas: Acolhimento: afeto, carinho, diálogo e confiança na relação com os profissionais de saúde; Acolhimento: resolutividade das demandas, acesso e acessibilidade ao serviço de saúde no contexto rural.

O estudo segue as diretrizes da Resolução nº. 466, de 12 de dezembro de 2012, do Conselho Nacional de Saúde, tendo sido aprovado em 9 de agosto de 2017 pelo Comitê de Ética em Pesquisa institucional, mediante o Parecer $n^{\circ}$ 2.208.566. A produção das informações foi realizada após leitura, explicação e assinatura do Termo de Consentimento livre e Esclarecido pelos participantes. Tendo em vista a garantia do anonimato, os depoimentos foram identificados pela letra " $P$ " referente a participante, seguida de número ordinal correspondente à ordem de participação na pesquisa.

\section{Resultados}


Em relação ao perfil sociodemográfico dos participantes, 11 eram do sexo masculino e dois do sexo feminino. A idade variou de 38 a 84 anos. Quanto ao nível de escolaridade, oito possuíam ensino fundamental incompleto; três, segundo grau completo; e dois, ensino fundamental completo. Em relação à renda, nove participantes mencionaram que esta era proveniente de aposentadoria por invalidez e da agricultura. Apenas quatro participantes tinham como fonte de renda exclusivamente o Benefício de Prestação Continuada (BPC). A seguir, serão discutidas as categorias temáticas que emergiram das entrevistas semiestruturadas.

Acolhimento: afeto, carinho, diálogo e confiança na relação com os profissionais de saúde

Esta categoria revela que os participantes relacionavam o acolhimento à demonstração de carinho e afeto. Assim, reportaram-se à forma como eram recebidos pelos profissionais de saúde das equipes de ESF.

Acolhimento é tu chegar [...] ser bem recebido, bem acolhido pelos profissionais de ambas as áreas [...] Médico, Enfermeiro, Técnico de Enfermagem, Auxiliar de Enfermagem, Farmacêutico. Isso aqui na unidade é nota 10 [...] meu vínculo com a unidade é muito grande, me sinto muito bem acolhido. (P6)

A gente é recebida muito bem [...] eu conheço dois doutores [se referindo aos médicos da equipe] os outros não [...] Ali no posto sou bem atendida [...]. (P9)

Pôde-se identificar por meio dos depoimentos dos participantes que existiam profissionais pelos quais se sentiam mais acolhidos e com os quais tinham maior proximidade. Eles destacaram enfermeiras, ACS e médicos.

Enfermeira e agente de saúde porque tem mais amizade, conhecimento.

Converso mais com a enfermeira do posto, conheço melhor [...]. (P4) 
Acolhimento na Estratégia Saúde da Família: perspectivas das pessoas com deficiência... 8

[...] a Enfermeira, porque tu chegas lá e conta o causo [se referindo à sua demanda] para ela [...] ela vai e verifica se tem ficha para te atender. (P5)

Eu gosto bastante do enfermeiro [...] a gente conhece mais, tem uma amizade [...] se eu vou ao posto eu prefiro consultar com ele [...] não que os outros não sejam bons, mas eu prefiro consultar com ele. (P8)

[...] eu tenho muita amizade com os doutores e as enfermeiras. (P9)

Eu gosto mais do médico $[. .].(\mathrm{P} 13)$

O acolhimento foi revelado pelos participantes pela liberdade que estes sentiam ao dialogar com os profissionais de saúde. Ao sentirem-se livres, conseguiam expressar suas demandas em relação ao processo saúde e adoecimento, bem como dúvidas e anseios.

Sim [se referindo ao diálogo com os profissionais] meus problemas, minhas dores, minhas doenças [...] me sinto à vontade. (P2)

Sempre me sinto muito à vontade para tirar dúvidas [...] nossa relação é muito boa. (P6)

Eu não escondo [...] eu não escondo o que eu sinto, eu falo [...] não tenho vergonha. (P7)

Um dos depoimentos evidencia que o diálogo se constitui como elemento fundamental no processo de cuidado. Este permite a aproximação entre os profissionais de saúde e as PCDs.

[...] esse médico é bem atencioso [...] tem gente que vai lá [referindo-se à unidade de saúde da família] só para conversar com ele porque ele tem paciência com as pessoas. $(\mathrm{P} 11)$

Esta categoria permitiu identificar que o acolhimento era percebido pelas PCDs como a forma que eram recebidas pelos profissionais das equipes de ESF e pela relação de diálogo e confiança estabelecida com estes. Nesta relação, os participantes também apontaram profissionais com posturas mais acolhedoras e com os quais estabeleceram um vínculo de maior proximidade. 
9 | Lenz TC, Costa MC, Colomé ICS, Andrade A, Souza NS, Arboit J

Acolhimento: resolutividade das demandas, acesso e acessibilidade ao serviço de saúde no contexto rural

A partir desta categoria, identifica-se que o acolhimento era relacionado pelas PCDs à resolutividade de suas demandas. Esta resolutividade ocorria a partir das condutas das próprias equipes de ESF ou a partir do encaminhamento para outros serviços.

[...] o problema de um jeito ou de outro, é resolvido [...] nunca deixei de ser atendido [...] nunca ninguém deixou de resolver o problema [...]. (P6)

[...] no posto de saúde dentro do possível, eles acabam resolvendo ali e se não conseguem eles mandam para outro lugar [referindo-se a outro município] [...] ou resolve ali ou eles dão um jeito de encaminhar. (P8)

[...] eu sou muito bem atendida e minhas coisas sempre são resolvidas [...] eu vou quando preciso consultar, pedir medicamento, renovar receita. (P10)

Sempre fui bem atendido [...] o médico sempre encaminha para repetir os exames. (P11)

[...] sempre fui bem atendido, sempre resolveram tudo [...]. (P12)

Apesar de alguns participantes destacarem o bom atendimento e a resolutividade das demandas apresentadas, outros depoimentos revelaram dificuldades para a garantia do acesso aos serviços. Estas se relacionavam, especialmente, com a indisponibilidade de exames gratuitos, falta de medicação, atendimento médico limitado e sistema de fichas para atendimento.

[...] às vezes tu tens a necessidade de fazer um exame ali e já não tem [...] eu estou com um raio- $X$ lá para fazer e está parado porque eu não tenho condições de pagar. (P3) 
Acolhimento na Estratégia Saúde da Família: perspectivas das pessoas com deficiência...| 10

[...] a gente chega lá, vai ao médico, pega a receita e não tem remédio [...] não são todas resolvidas [referindo-se às demandas de cuidado] algumas tem que resolver por conta. (P5)

[...] nunca tem remédio, nunca pude pegar um remédio ali toda vida [...] médico nunca consigo também porque tem que ir às cinco da manhã pegar uma ficha para poder pegar uma receita [...] e ainda, quando tem médico porque agora acho que nem está tendo [...] diz que vem uma médica nova para atender e atende sete fichas por dia [...] o que adianta sete fichas numa população [...] então para mim nem adianta ir no posto. (P7)

É que tem que estar cedo lá para conseguir ficha, e eles têm só 16 fichas e eu era 17 então não consegui [...] e daí tem que chegar cedo, tudo por ordem de chegada. (P10)

As PCDs também expuseram desafios no que tange à acessibilidade aos serviços de saúde. Nos municípios nos quais não havia unidade de ESF no contexto rural, para chegar até este serviço, as PCDs física necessitavam acionar a Secretaria de Saúde e solicitar transporte, que muitas vezes demorava.

[...] às vezes o carro da saúde que a gente chama demora demais [...] (P2)

Em outros municípios, embora existissem unidades de ESF nas comunidades rurais, estas tinham limitações importantes para a acessibilidade das PCDs. Dentre elas, se encontravam a ausência de corrimão e rampas.

Corrimão não tem [...] é acesso rente com o chão [...] chovendo, resvala as muletas [...] tem que entrar uma pessoa me segurando atrás porque ela é lisa, derrapante. (P5)

A cadeira de roda não [...] eu chego ali, subo até lá de carro, daí abrem a cadeira, me pesam e me colocam na cadeira [...] tem que ir, mas na maioria das vezes eu fico no carro [...] o médico vem ali [referindo-se ao carro] (P13) 
Nesta categoria, as PCDs revelaram o acolhimento, relacionando-o à resolutividade de suas demandas, as quais, em sua maioria, eram atendidas. Por outro lado, apontaram algumas dificuldades de acesso e acessibilidade aos serviços de saúde.

\section{Discussão}

Para as PCDs, o acolhimento da equipe de ESF remete às relações de carinho e afeto, o que não destoa da perspectiva de acolhimento, segundo a qual seu propósito volta-se dentre outros, para a escuta qualificada e o compromisso de ofertar um cuidado competente, de qualidade e resolutivo. ${ }^{12}$ Os participantes referiam que serem bem recebidos pelos profissionais também se caracteriza como acolhimento. Tal percepção foi igualmente evidenciada por estudo de revisão integrativa da literatura, segundo o qual as percepções dos usuários permitiram constatar que estes relacionavam o acolhimento à boa receptividade pela equipe. ${ }^{13}$

No processo de cuidado, muitos eixos são consideráveis para o acolhimento ser efetivo, com destaque para as relações de proximidade entre o profissional que realiza o acolhimento e o usuário. ${ }^{12}$ Nos depoimentos, os participantes relataram a importância de manter relações de vínculo com os profissionais, com destaque para as enfermeiras, ACS e médicos, com a justificativa de que estes profissionais fomentavam a construção de relações de amizade, o estabelecimento de diálogo e a resolutividade de suas demandas em saúde. Nesta perspectiva, citaram o espaço da consulta com o enfermeiro, o que remete à percepção de que a consulta pode implicar o acolhimento.

O estabelecimento de relações de vínculo e responsabilização entre a equipe da Atenção Primária e a comunidade adscrita no território é destacado como elemento que permeia o processo de trabalho neste nível de atenção à saúde. ${ }^{14} \mathrm{O}$ vínculo é um processo de aproximação que gera laços afetivos entre dois seres humanos: o profissional de saúde capacitado para o cuidado e o usuário que 
Acolhimento na Estratégia Saúde da Família: perspectivas das pessoas com deficiência...| 12

procura atendimento para as suas necessidades de cuidado. ${ }^{8}$ Este processo é construído a partir de posturas acolhedoras, de abertura aos problemas do outro e na relação de empatia.

Ademais, o vínculo articula conceitos de humanização, responsabilização, acolhimento e integralidade. Desse modo, possibilita que os profissionais conheçam além das necessidades de saúde, o contexto em que usuário e famílias vivem ${ }^{15}$, reconhecendo assim, seus determinantes sociais e singularidades.

Em se tratando do estabelecimento de vínculo entre profissional e usuário, os ACS são aqueles que possuem maior proximidade com os entrevistados, uma vez que estes estabelecem maior contato com as famílias e comunidade, tanto no contexto urbano, quanto rural. Contudo, neste último, considerando as especificidades da distância geográfica em relação às unidades de ESF e outros serviços de saúde, o papel do ACS se torna ainda mais importante, pois, muitas vezes, é este o primeiro profissional de saúde que o usuário aciona quando possui alguma demanda.

Ao morarem no território em que atuam, encontram-se disponíveis para sua comunidade tanto em horário de expediente, quanto fora dele. As práticas do ACS envolvem ações de promoção e vigilância em saúde, como a busca ativa. ${ }^{16}$ Seu processo de trabalho se dá a partir de um amplo escopo pautado em relações assistenciais e de vínculo. ${ }^{17}$

O diálogo foi outro elemento referido pelos participantes, pela possibilidade de terem liberdade para expressar suas queixas durante o atendimento, sem sentirem vergonha dos seus problemas. Este resultado é satisfatório e positivo, tanto para a PCD, que se sente livre e à vontade para dialogar, assim como para o profissional, que, a partir desta liberdade de expressão e diálogo, consegue traçar um plano terapêutico que atenda às demandas deste usuário. Nessa direção, destaca-se que a qualidade da comunicação profissional-usuário associada a um vínculo sólido e à sensibilidade do profissional para identificar o contexto do usuário em sua plenitude, promove uma atenção integral à saúde. ${ }^{18}$ 
No que tange à resolutividade das demandas das PCDs física residentes no contexto rural, pôde-se inferir que esta ocorre de forma parcial. Nesse sentido, alguns participantes relataram a falta de medicação disponível, o atendimento médico reduzido a um número limitado de fichas, impossibilidade de realização de exames gratuitamente e agendamento de atendimento por fichas.

Os depoimentos dos participantes que associaram o acolhimento à resolutividade de demandas de cunho essencialmente biológico, como realização de exames e acesso a medicamentos revelam a hegemonia do modelo biomédico, curativo e biologicista. Esse modelo, além de ser reproduzido em muitas práticas de atenção à saúde pelos profissionais, também acaba sendo incorporado pelos usuários, neste caso, as PCDs física, quando estes buscam atenção às suas demandas em saúde. Neste contexto, a Política Nacional de Saúde da Pessoa Portadora de Deficiência no âmbito do SUS aponta a relevância do atendimento de suas demandas, sejam elas relacionadas ou não à deficiência que possuem, buscando a integralidade da atenção à saúde. ${ }^{3}$ Isso será possível se for superada a ótica que privilegia o reconhecimento da PCD apenas por sua condição biológica.

Alguns participantes relataram a necessidade de agendamento por meio de fichas, que possuíam limite diário no cenário em estudo. Além disso, para conseguir atendimento via fichas, precisavam chegar muito cedo à unidade e, mesmo assim, por vezes, não obtinham sucesso. Ao considerar que, do total de municípios cenário do estudo em tela, apenas dois contavam com unidades de ESF no contexto rural, as dificuldades de acesso para as PCDs se agravam, pelo tempo de deslocamento do contexto rural até o urbano. Dificuldades de PCDs física residentes em áreas rurais para acessar serviços de saúde foram relatadas também em estudo desenvolvido no Peru, sendo maiores do que no cenário urbano. ${ }^{19}$

No Brasil, a APS, por meio da ESF, tem evoluído em relação à cobertura populacional, buscando romper com o modelo médico assistencial e constituir-se porta de entrada 
Acolhimento na Estratégia Saúde da Família: perspectivas das pessoas com deficiência...| 14

preferencial ao SUS. ${ }^{20}$ No entanto, a garantia do acesso à saúde ainda não se concretizou para uma grande parte da população brasileira, dentre esta, a que reside no contexto rural. ${ }^{21}$

Tais reflexões revelam a premência da revisão de estratégias de atendimento e cuidado, especialmente quando os usuários residem no contexto rural e possuem alguma deficiência, de modo a proporcionar o acesso a esses usuários, sendo uma destas o atendimento da demanda espontânea. Nessa direção, pontua-se que este se refere à oferta do serviço de saúde, possibilitando a sua utilização apropriada para alcançar os melhores resultados de saúde. ${ }^{22} \mathrm{Na}$ medida em que a restrição do número de fichas não permite o atendimento dos usuários, o acesso à saúde é prejudicado.

Considerando as limitações de acesso da população rural aos serviços de saúde, seria importante que as equipes de ESF buscassem romper com programações inflexíveis e passassem a atender à demanda espontânea e no mesmo dia de comparecimento destes usuários à unidade. Isso demonstraria maior sensibilidade às demandas da população rural, que tende a enfrentar longas distâncias para chegar até o serviço de saúde. ${ }^{21}$

Apesar das limitações, os entrevistados reconheceram e valorizaram o esforço que a equipe de ESF empreendia em acolhê-los e buscar a resolução de suas demandas. Estes achados vão ao encontro de estudo que objetivou compreender a satisfação dos usuários com o acesso e o acolhimento da APS em Ribeirão Preto/São Paulo, Brasil. Este revelou que, embora os usuários tenham apontado fragilidades na atenção à saúde, como demora no agendamento de consultas e no atendimento de demandas, eles avaliaram de modo positivo o acolhimento, atenção e diálogo proporcionados pelos profissionais da APS. ${ }^{23}$

Em dois municípios, embora houvesse unidades de ESF no contexto rural, estas não possibilitavam a acessibilidade adequada às PCDs física devido à infraestrutura inadequada, tanto interna quanto externa, da unidade. Os depoimentos revelaram que esta infraestrutura impossibilitava a acessibilidade de PCDs em cadeira de rodas, já que uma destas teve que ser 
atendida no carro. Ainda, sugeriram que o piso de material liso dificultava o uso de muletas, e com isso as PCDs necessitavam de ajuda de outras pessoas, diminuindo sua autonomia. Na direção destes achados, estudo que objetivou avaliar a acessibilidade física da recepção de unidades APS e sua relação com o acolhimento identificou que rampas de acesso e piso foram alguns itens que possuíam maior probabilidade de serem inacessíveis na área rural do que na urbana. ${ }^{24}$

Considera-se acessibilidade a possibilidade que o usuário possui ou não para chegar ao serviço de saúde. No estudo em tela acesso e acessibilidade são considerados conceitos complementares. ${ }^{22}$ Assim, na medida em que as PCDs possuem dificuldades de acessibilidade ao serviço de saúde, seu acesso à saúde também é prejudicado/limitado.

Sob essa perspectiva, estudo desenvolvido em Bangladesh revelou que as PCDs física, enfrentaram barreiras relacionadas à mobilidade para acessar serviços de APS, inclusive requerendo o auxílio de outras pessoas para chegar a estes serviços. ${ }^{6}$ Para a promoção da acessibilidade, as unidades de saúde precisam possuir adaptações ambientais condizentes com as necessidades das PCDs, contribuindo para a sua autonomia e independência, sejam totais ou assistidas. ${ }^{25}$

Ainda em relação à infraestrutura, estudo quantitativo desenvolvido na Índia concluiu que rampas, corredores e cadeiras de rodas, são algumas das barreiras à acessibilidade de PCD em centros de APS, os quais não estão equipados de forma adequada para assistir essas pessoas. ${ }^{26}$ Em investigação qualitativa buscando explorar as perspectivas dos profissionais de saúde na prestação de serviços de APS para PCD física na zona rural de Gana, estes, em sua maioria, apontaram que as instalações inacessíveis constituíam um importante obstáculo para a prestação de serviços a usuários com deficiências físicas. Revelaram ainda que as unidades foram projetadas sem considerar as necessidades de pessoas que utilizam dispositivos de assistência, como cadeira de rodas. ${ }^{27}$

As dificuldades de acessibilidade aos serviços também, são reveladas pela carência de transporte fornecido pelo setor da saúde, que, muitas vezes, é demorado, além da necessidade de 
Acolhimento na Estratégia Saúde da Família: perspectivas das pessoas com deficiência...| 16

deslocamento a outro município para realizar exames de saúde. Estes dados desvelam o cotidiano das pessoas que residem em contextos rurais, permeado por entraves como a distância geográfica e a falta de transporte. A estes entraves se somam os limites que a deficiência física impõe como a dificuldade de mobilidade.

A acessibilidade dos usuários aos serviços de saúde e seus impactos no acesso dos usuários ainda, configuram um desafio para o SUS, mesmo após 32 anos de implantação dessa política pública de saúde. Ao considerar o contexto rural e as particularidades das PCDs física, os desafios de acessibilidade tendem a se acentuar. Tais desafios dificultam a integralidade em saúde e contribuem para sustentar as iniquidades a que estas pessoas estão submetidas. ${ }^{28}$

No Brasil, o processo de democratização da saúde a partir do SUS tem contribuído para a inclusão das PCDs nas políticas públicas de saúde. ${ }^{29}$ No entanto, o panorama apresentado no estudo em tela reforça que a saúde destas permanece inserida em uma agenda pouco acolhida neste Sistema, desconsiderando além do princípio da integralidade, os princípios da equidade e acesso universal à saúde. ${ }^{30}$

O estudo apresenta limitações como o número restrito de participantes de um contexto rural específico, porém traz como contribuição subsídios para se pensar novas práticas de inclusão e acessibilidade das PCDs física que vivem no contexto rural aos seus direitos, em especial, o direito à saúde. Dentre essas práticas, pontua-se o atendimento dessas pessoas a partir da demanda espontânea, proporcionando acolhimento de suas necessidades quando estas buscam o serviço de APS. Ainda, sinaliza-se a readequação da infraestrutura das unidades de ESF a fim de proporcionar a acessibilidade das PCDs física mediante rampas e corrimão.

\section{Conclusão}

Os achados do estudo apresentam diferentes elementos que corroboram com o referencial de acolhimento, visto que as PCDs física residentes no contexto rural o remetem a 
demonstrações de carinho, afeto e a uma relação de confiança e proximidade com os profissionais e com o atendimento da equipe. Nessa direção, se sentiam mais acolhidas especialmente pelo enfermeiro, ACS e médico.

O acolhimento também foi apontado pelos participantes como a possibilidade de dialogar abertamente com os profissionais sobre as suas necessidades de saúde. Ainda, foi relacionado à resolutividade imediata de suas demandas pelas equipes ou encaminhamento para outros serviços. Apesar disso, alguns participantes citaram que nem sempre estas demandas eram atendidas, especialmente em relação à disponibilidade de medicações, exames, atendimento médico e agendamento do atendimento pelo sistema de fichas, o que revela a predominância do modelo biomédico em seu ideário.

Os relatos dos participantes também mostraram que o acolhimento se relaciona à acessibilidade aos serviços de saúde. Embora, em alguns municípios, houvesse unidades de ESF nos contextos rurais, sua infraestrutura interna e externa dificultava e/ou impossibilitava o acesso das PCDs física, em especial aquelas que utilizavam cadeira de rodas e muletas.

Os resultados contêm elementos relevantes para subsidiar gestores locais de saúde e profissionais dos serviços de Atenção Primária à Saúde, no sentido de estruturar as agendas locais, considerando as particularidades do contexto rural, a fim de proporcionar o acolhimento das PCDs física em sua integralidade. Assim, é imperativo considerar suas percepções e representações como ferramentas para a formulação de ações pelo setor da saúde, contemplando as garantias institucionais previstas nos princípios e diretrizes do SUS, e, principalmente, buscando a concretização da saúde como direito de cidadania.

\section{Referências}

1. Sisti DA. Naturalism and the social model of disability: allied or antithetical? J Med Ethics. 2015;41:553-6. doi: 10.1136/medethics-2014-102127 
2. Bampi LNS, Guilhem D, Alves ED. Social model: a new approach of the disability theme. Rev Latinoam Enferm. 2010;18(4):816-23. doi: 10.1590/S0104-11692010000400022

3. Ministério da Saúde (BR), Secretaria de Atenção à Saúde. Política nacional de saúde da pessoa portadora de deficiência [Internet]. Brasília (DF): Ministério da Saúde; 2008 [acesso em 2019 fev 20]. Disponível em: http://bvsms.saude.gov.br/bvs/publicacoes/politica_nacional_saude_pessoa_deficiencia.pdf

4. Holanda CMA, Andrade FLJP, Bezerra MA, Nascimento JPS, Neves RF, Alves SB, et al. Support networks and people with physical disabilities: social inclusion and access to health services. Ciênc Saúde Colet. 2015;20(1):175-84. doi: 10.1590/1413-81232014201.19012013

5. Bortolotto CC, Mola CL, Tovo-Rodrigues L. Quality of life in adults from a rural area in Southern Brazil: a population-based study. Rev Saúde Pública. 2018;52(1):1-11. doi: 10.11606/s15188787.2018052000261

6. Talukdar JR, Mahmud I, Rashid SF. Primary health care seeking behaviour of people with physical disabilities in Bangladesh: a cross-sectional study. Arch Public Health. 2018;76(43). doi: 10.1186/s13690018-0293-1

7. Alves EAB, Silva MT. Processo de trabalho em saúde: estudo da relação entre recursos imateriais e resolutividade. Rev Espacios [Internet]. 2015 [acesso em 2020 fev 16];36(16):1-12. Disponível em: https://www.revistaespacios.com/a15v36n16/15361612.html

8. Ministério da Saúde (BR), Secretaria de Atenção à Saúde, Núcleo Técnico da Política Nacional de Humanização. HumanizaSUS: documento base para gestores e trabalhadores do SUS [Internet]. Brasília (DF): Ministério da Saúde; 2006 [acesso em 2019 jul 08]. Disponível em: https://bvsms.saude.gov.br/bvs/publicacoes/documento_base.pdf

9. Lopes AS, Vilar RLA, Melo RHV, França RCS. O acolhimento na Atenção Básica em saúde: relações de reciprocidade entre trabalhadores e usuários. Saúde Debate. 2015;39(104):114-23. doi: 10.1590/0103110420151040563

10. Silva TF, Romano VF. Sobre o acolhimento: discurso e prática em Unidades Básicas de Saúde do município do Rio de Janeiro. Saúde Debate. 2015;39(105):363-74. doi: 10.1590/0103-110420151050002005

11. Minayo MCS. O desafio do conhecimento: pesquisa qualitativa em saúde. $14^{\mathrm{a}}$ ed. São Paulo: Hucitec; 2014.

12. Ministério da Saúde (BR), Secretaria de Atenção à Saúde, Núcleo Técnico da Política Nacional de Humanização. Acolhimento nas práticas de produção de saúde [Internet]. Brasília (DF): Ministério da Saúde; 2010 [acesso em 2020 fev 24]. Disponível em: https://bvsms.saude.gov.br/bvs/publicacoes/acolhimento_praticas_producao_saude.pdf 
13. Coutinho LRP, Barbieri AR, Santos MLM. Acolhimento na Atenção Primária à Saúde: revisão integrativa. Saúde Debate. 2015;39(105):514-24. doi: 10.1590/0103-110420151050002018

14. BRASIL. Ministério da Saúde. Portaria no 2.436, de 21 de setembro de 2017. Aprova a Política Nacional de Atenção Básica, estabelecendo a revisão de diretrizes para a organização da Atenção Básica, no âmbito do Sistema Único de Saúde (SUS). Brasília, DF, Ministério da Saúde, 2017. Disponível em: http://bvsms.saude.gov.br/bvs/saudelegis/gm/2017/prt2436_22_09_2017.html. Acesso em: 8 jul. 2019.

15. Santos RCA, Miranda FAN. Importância do vínculo entre profissional-usuário na estratégia de saúde da Família. Rev Enferm UFSM. 2016;6(3):350-9. doi: 10.5902/2179769217313

16. Barreto ICHC, Pessoa VM, Sousa MFA, Nuto SAS, Freitas RWJF, Ribeiro KG, et al. Complexidade e potencialidade do trabalho dos Agentes Comunitários de Saúde no Brasil contemporâneo. Saúde Debate. 2018;42(N Esp 1):114-29. doi: 10.1590/0103-11042018s108

17. Pinto AGA, Palácio MAV, Lôbo AC, Jorge MSB. Vínculos subjetivos do agente comunitário de saúde no território da Estratégia Saúde da Família. Trab Educ Saúde. 2017;15(3):789-802. doi: 10.1590/1981-7746sol00071

18. Assunção GS, Queiroz E. Abordagem do tema "relação profissional de saúde-paciente" nos cursos de saúde da universidade de Brasília. Psicol Ensino Form [Internet]. 2015 [acesso em 2020 fev 24];6(2):18-36. Disponível em: http://pepsic.bvsalud.org/pdf/pef/v6n2/v6n2a03.pdf

19. Moscoso-Porras M, Fuhs AK, Carbone A. Access barriers to medical facilities for people with physical disabilities: the case of Peru. Cad Saúde Pública. 2019;35(12):e00050417. doi: 10.1590/0102311X00050417

20. Pessoa VM, Almeida MM, Carneiro FF. Como garantir o direito à saúde para as populações do campo, da floresta e das águas no Brasil? Saúde Debate. 2018;42(N Esp 1):302-14. doi: 10.1590/010311042018 s 120

21. Garnelo L, Lima JG, Rocha ESC, Herkrath FJ. Acesso e cobertura da Atenção Primária à Saúde para populações rurais e urbanas na região norte do Brasil. Saúde Debate. 2018;42(N Esp 1):81-99. doi: 10.1590/0103-11042018S106

22. Starfield B. Atenção primária: equilíbrio entre necessidades de saúde, serviços e tecnologia. Brasília (DF): Unesco, Ministério da Saúde; 2002.

23. Gomide MFS, Pinto IC, Bulgarelli AF, Santos ALP, Gallardo MPS. User satisfaction with primary health care: an analysis of access and care. Interface Comun Saúde Educ. 2018;22(65):387-98. doi: 10.1590/1807-57622016.0633

24. Marques JF, Áfio ACE, Carvalho LV, Leite SS, Almeida PC, Pagliuca LMF. Physical accessibility in primary healthcare: a step towards the embracement. Rev Gaúch Enferm.2018;39:e2017-0009. doi: 
Acolhimento na Estratégia Saúde da Família: perspectivas das pessoas com deficiência...| 20

https://doi.org/10.1590/1983-1447.2018.2017-0009

25. Martins KP, Costa TF, Medeiros TM, Fernandes MGM, França ISX, Costa KNFM. Internal structure of Family Health Units: access for people with disabilities. Ciênc Saúde Colet. 2016;21(10):3153-60. doi: 10.1590/1413-812320152110.20052016

26. Nischith KR, Bhargava M, Akshaya KM. Physical accessibility audit of primary health centers for people with disabilities: an on-site assessment from Dakshina Kannada district in Southern India. J Family Med Prim Care. 2018;7(6):1300-03. doi: 10.4103/jfmpc.jfmpc_177_18

27. Dassah E, Aldersey HM, McColl MA, Davison C. Healthcare providers' perspectives of providing primary healthcare services to persons with physical disabilities in rural Ghana. Prim Health Care Res Dev. 2019;20(e108):1-8. doi: 10.1017/S1463423619000495

28. Amorim EG, Liberali R, Medeiros Neta OM. Avanços e desafios na atenção à saúde de pessoas com deficiência na Atenção Primária no Brasil: uma revisão integrativa. Holos (Natal Online). 2018;34(1):22436. doi: 10.15628/holos.2018.5775

29. Fiorati RC, Elui VMC. Social determinants of health, inequality and social inclusion among people with disabilities. Rev Latinoam Enferm. 2015;23(2):329-36. doi: 10.1590/0104-1169.0187.2559

30. Campos MF, Souza LAP, Mendes VLF. A rede de cuidados do Sistema Único de Saúde à saúde das pessoas com deficiência. Interface Comun Saúde Educ. 2015;19(52):207-10. doi: 10.1590/180757622014.0078

Editor Científico: Tânia Solange Bosi de Souza Magnago

Editor associado: Carine Vendruscolo

Fomento: Trabalho financiado pela Chamada FAPERGS/MS/CNPQ/SESRS n. 03/2017 Programa pesquisa para o SUS: gestão compartilhada em saúde PPSUS - 2017.

\section{Autor correspondente}

Marta Cocco da Costa

E-mail: marta.c.c@ufsm.br

Endereço: Av. Independência, 3751, Bairro Vista Alegre, Palmeira das Missões (RS), Brasil

CEP: $98300-000$

\section{Contribuições de autoria}

1 - Taís Cristiane Lenz 
Concepção ou desenho do estudo/pesquisa; análise e/ou interpretação dos dados; revisão final com participação crítica e intelectual no manuscrito.)

\section{2 - Marta Cocco da Costa}

Concepção ou desenho do estudo/pesquisa; análise e/ou interpretação dos dados; revisão final com participação crítica e intelectual no manuscrito.

\section{3 - Isabel Cristina dos Santos Colomé}

Análise e/ou interpretação dos dados; revisão final com participação crítica e intelectual no manuscrito.

\section{4 - Andressa de Andrade}

Análise e/ou interpretação dos dados; revisão final com participação crítica e intelectual no manuscrito.

\section{5 - Neila Santini de Souza}

Análise e/ou interpretação dos dados; revisão final com participação crítica e intelectual no manuscrito.

\section{6 - Jaqueline Arboit}

Análise e/ou interpretação dos dados; revisão final com participação crítica e intelectual no manuscrito.

\section{Como citar este artigo}

Lenz TC, Costa MC, Colomé ICS, Andrade A, Souza NS, Arboit J. Acolhimento na saúde da família - perspectivas das pessoas com deficiência que residem no rural. Rev. Enferm. UFSM. 2021 [Acesso em: Mês Dia Ano]; vol.11 e3: 1-21. DOI:https://doi.org/10.5902/2179769244155 